\title{
Cerebellar Regulation of Motor Timing and Coordination
}

\author{
Yifat Prut
}

\begin{abstract}
In human and non-human primates, motor timing is considered to be dictated by cerebellar control of motor cortical activity, relayed through the cerebellarthalamo-cortical (CTC) system. This supposition relies on studies that documented motor impairments in cerebellar patients and in animal models. However, we know very little about the way cerebellar information is integrated by cortical circuitry to affect motor cortical commands. We addressed this question by probing the CTC system in a primate model. We found that a brief activation of this pathway efficiently recruits motor cortical cells throughout the motor and premotor cortex. However, cortical response was dominated by a powerful inhibition that truncated the early excitation. At movement onset, CTC input transiently synchronized neighboring neurons. Blocking the CTC pathway produced motor impairments similar to the symptoms of cerebellar ataxia. The motor deficits were preceded by changes in neural activity that included local and global desynchronization. It is suggested that the excitation-inhibition interplay triggered by CTC input shapes the response profile of a distributed network of motor cortical neurons as required to initiate and coordinate movements.
\end{abstract}

\footnotetext{
Y. Prut $(\otimes)$

Department of Medical Neurobiology, IMRIC ELSC, The Hebrew University, Jerusalem, Israel e-mail: yifatpr@ekmd.huji.ac.il
} 